\title{
Variabilité de la croissance et de la qualité chez la pêche (Prunus persica L Batsch) et liaison entre croissance et qualité
}

\author{
M Génard 1, C Bruchou 2, M Souty 3 \\ 1 INRA, agronomie; \\ 2 INRA, biométrie; \\ ${ }^{3}$ INRA, technologie des produits végétaux, BP 91, domaine Saint-Paul, 84143 Montfavet Cedex, France
}

(Reçu le 21 mars 1991; accepté le 6 octobre 1991)

\begin{abstract}
Résumé - La croissance de la pêche a été décrite par un modèle de croissance déterministe à 2 phases. Les courbes de croissance cumulée et de vitesse de croissance de 120 fruits ont été comparées par analyse en composantes principales sur les paramètres du modèle. Les différences entre fruits sont importantes pendant la deuxième phase de croissance. Après récolte, les qualités gustative (composition biochimique) et commerciale (fermeté et coloration) de chaque fruit ont été analysées. Le pH est peu variable; l'acidité, l'indice réfractométrique et la teneur en sucres forment un ensemble de descripteurs intercorrelés qui séparent les fruits selon un gradient de qualité gustative et de fermeté auquel est liée la coloration jaune. Les autres paramètres de la coloration sont liés entre eux et sont non corrélés au groupe précédent. La relation entre croissance et qualité a été analysée par analyse factorielle des correspondances sur variables instrumentales. Les fruits mûrs précocement et ceux ayant un diamètre élevé à l'issue de la première phase ont des taches de couleur rouge violacé alors que l'orange et une chrominance plus élevée caractérisent les autres fruits. Les fruits ayant une deuxième phase de croissance importante et caractérisés par une vitesse maximale de croissance élevée et tardive sont plus sucrés, moins acides et ont une fermeté plus faible. La variation de la luminance n'est pas liée à celle de la croissance.
\end{abstract}

Prunus persica $=$ pêcher $/$ qualité $/$ fruit $/$ modèle de croissance $/$ analyse multivariée

Summary - Variability of peach (Prunus Persica L Batsch) growth and quality, and relationships between growth and quality. The individual fruit growth of the peach (Suncrest cultivar), was studied by modelling growth curves based on cheek-diameter measurements. Our model assumed that peach fruit growth; was divided into 2 phases. The first one described the pit growth and the first part of the flesh growth; the second described the second part of the flesh growth (fig 1). The fit of the model was good (fig 2). The growth curves were compared using a particular principal component analysis (PCA) working on the model parameters. The differences between fruits were highest during the second phase (fig 3). The curves were grouped into classes using a cluster analysis running on PCA factors (fig 4). At harvest, quality data, ie $\mathrm{pH}$, soluble solids, sucrose, reducing sugars, citric and malic acids, flesh firmness and skin color, were recorded. Quality variation among fruits was studied using a correspondence analysis and a cluster analysis. The $\mathrm{pH}$ did not vary (table I). Acidity, sugars and soluble solids were correlated and described a gustatory gradient (fig 5). Firmness and yellow color were correlated with this gradient. Other color descriptors were correlated to each other and described a coloration gradient which was independent from the gustatory one (fig 6). Four groups of peach fruits were individualized (figs 5, 6). For most quality descriptors, between-class differences were high (table II). The relationship between growth and quality was studied using a canonical correspondence analysis. The peach diameter in April was not correlated with quality (table IV). Growth was more correlated with gustatory quality and firmness than with coloration (tables I and III). Early mature fruits with a high cheek diameter at the end of the first phase had a large amount of purple. The other fruits had a large amount of orange and a high chrominance (fig 9). In fruits with both a significant second growth phase and a high and late maximal growth rate the highest soluble solids and sucrose content and the lowest citric acid content and firmness were found (fig 8). Luminance was not correlated with growth. 


\section{INTRODUCTION}

La qualité d'un produit se définit en fonction de ses différentes utilisations. Chez le fruit, on parlera ainsi de qualité commerciale (résistance aux manipulations, aptitude à la conservation, aspect), de qualité gustative (goût, texture), de qualité sanitaire, etc, chacune étant caractérisée par de nombreux critères. Divers travaux montrent qu'il existe une importante variabilité des valeurs de ces critères selon la situation du fruit dans l'arbre (Marini et Trout, 1984; Dann et Jerie, 1988; Monestiez et al, 1989). Cette variabilité peut être notamment expliquée par des différences de condition d'ensoleillement (Doud et Ferree, 1980; Erez et Flore, 1986; Patten et al, 1986), de situation vis-à-vis des feuilles productrices d'assimilats (Magness, 1928; Weinberger et Cullinan, 1932; Roper et Loescher, 1987) et dans le nombre d'organes compétiteurs pour ces assimilats (Kaps et Cahoon, 1989; Reynolds, 1989). La liaison souvent observée entre le calibre du fruit à la récolte et des critères de qualité tels que la teneur en sucres et l'acidité (Overholser et Claypool, 1931; Weinberger, 1931; Gaillard et al, 1976), montre que la qualité est également liée à la croissance du fruit. Il est nécessaire d'acquérir une meilleure connaissance de cette relation entre croissance et qualité du fruit dans une optique de modélisation de l'élaboration de la qualité. En effet, elle intègre l'action des facteurs de l'environnement cités précédemment auxquels la croissance est également sensible (Jones, 1931; Lai et al, 1990; Proebsting, 1990) et l'action directe du phénomène de croissance sur la qualité du fruit.

Notre objectif a été de décrire chez la pêche la variabilité de la croissance et de la qualité individuelle de fruits et de rechercher si les variations de qualité observées pouvaient être dues à des variations de croissance des fruits. Nous avons limité dans ce qui suit la notion de qualité à la qualité commerciale, définie par la fermeté et la coloration du fruit, et à la qualité gustative définie principalement par l'équilibre entre les sucres et l'acidité.

Dans une première étape nous avons décrit la croissance individuelle des pêches par modélisation des courbes de croissance. La comparaison des paramètres associés aux courbes de croissance individuelles a ensuite permis de dresser une typologie de ces courbes. Les principaux axes de variation de la qualité individuelle des fruits ont ensuite été étudiés, puis mis en relation avec la typologie précédente.

\section{MATÉRIEL ET MÉTHODES}

\author{
Matériel végétal
}

Les fruits proviennent de 12 pêchers de la variété Suncrest greffée sur GF677 situés dans un verger du centre INRA d'Avignon. Les pêchers étaient âgés de 8 ans et le mode de conduite était le gobelet. La variabilité des conditions locales de croissance dans l'arbre étant très importante, nous nous sommes limités à l'étude de la variabilité de la croissance et de la qualité d'une population de fruits occupant les rameaux orientés au sud et situés dans la partie supérieure des arbres. Quatre rameaux ont été échantillonnés en avril 1989 sur chaque arbre. Les rameaux ont été éclaircis et les fruits restants avaient en début d'étude des diamètres compris entre 14 et $21 \mathrm{~mm}$. Cent vingt fruits ont été étudiés pour leur croissance et 101 ont fait l'objet d'analyses de qualité.

\section{Mesures de la croissance et la qualité}

La croissance diamétrale des fruits a été mesurée hebdomadairement du 28 avril à la récolte (26 juillet au 17 août selon les fruits), à l'équateur du fruit et perpendiculairement à sa suture. Nous avons jugé que le fruit était mûr lorsque la croissance avait cessé, le fruit mollissait et avait une couleur de fond jaune orangée et non pas verte.

La mesure de la coloration a concerné la couleur de l'épiderme des fruits. Par analyse d'image (logiciel microscale, Holyoake, 1989) nous avons caractérisé 4 couleurs (jaune, orange, rouge, rouge violacé) et calculé leur taux de recouvrement, exprimé en pourcentage de la surface du fruit. La couleur de la face la plus rouge ou violacée a été en outre exprimée dans l'espace $L^{*}, a^{*}, b^{*}$ de la CIE (Delwiche et Baumgardner, 1983) après que les données spectrales aient été mesurées grâce à un spectrocolorimètre Hunterlab modèle Colorquest $C Q 1200 \mathrm{X} . \mathrm{L}^{*}$ représente la luminance $\left(L^{*}=0\right.$ pour un objet noir et $L^{*}=100$ pour un objet blanc); $a^{\star}$ et $b^{*}$ représentent la chrominance $\left(a^{*}\right.$ $=-60$ pour le vert et +60 pour le rouge; $b^{*}=-60$ pour le bleu et +60 pour le jaune). La fermeté des faces colorées et non colorées a été mesurée au moyen d'un pénétromètre à ressort avec un embout de $6 \mathrm{~mm}$ de diamètre.

Seuls les sucres totaux sont classiquement mesurés dans les études de qualité gustative. Cependant, de façon à mieux décrire les mécanismes en jeu dans l'élaboration de la qualité, nous avons considéré indépendamment le saccharose, sucre le plus abondant chez la pêche (Souty et André, 1975) et les sucres réducteurs dont les teneurs dans le fruit évoluent différemment au cours de la croissance. Les sucres totaux et réducteurs (exprimés en $\mathrm{g} / 100 \mathrm{~g}$ de matière fraîche) ont été dosés par la méthode à la nécuproïne (Bittner et Manning, 1967), la teneur en saccharose étant ob- 
tenue par différence entre ces 2 valeurs. L'acidité chez la pêche est surtout due aux acides malique et citrique, les fruits à faible rapport acide malique/acide citrique ayant une saveur plus acide (Souty et André, 1975). Les acides malique et citrique (exprimés en meq/100 g de matière fraîche) ont été obtenus par les méthodes de dosage enzymatique (Boehringer, 1988); ces dosages ont été réalisés au moyen d'un analyseur à flux continu. Nous avons également mesuré le $\mathrm{pH}$ qui exprime le degré d'ionisation de la partie des acides présents dans le fruit qui est non salifiée. II est donc une mesure de l'acidité du fruit en début de consommation. Lors de la consommation, l'acidité peut évoluer à la suite de la dissolution de la partie salifiée des acides. Enfin, nous avons évalué la teneur en matières solubles dans le fruit par la mesure de l'indice réfractométrique (IR). Cet indice est en général bien corrélé avec les notes de qualité données par les dégustateurs (Crochon, 1985). Un total de 16 variables de qualité a ainsi été étudié.

\section{Modélisation de la croissance}

La courbe de croissance de la pêche est une double sigmoïde qui peut être divisée en 4 périodes (Monet, 1983) et dont l'interprétation est présentée par Zuconi (1986). La première est une période de multiplication et d'élongation cellulaire du noyau et de la pulpe; le noyau atteint son diamètre final à l'issue de cette période. Lors de la deuxième période, la croissance est faible et il y a durcissement du noyau. La troisième période est caractérisée par une élongation importante des cellules de la pulpe, qui se stabilise lors de la quatrième période. Nous avons considéré avec Baker et Davis (1951) et Dejong et Goudrian (1989) que les différentes périodes décrites ci-dessus pouvaient être la résultante de 2 phases successives de développement du fruit. La première phase qui correspond à l'essentiel de la croissance jusqu'à mi-juin, est caractérisée par la croissance du noyau et la croissance précoce de la pulpe. Elle est tronquée à son début car nos mesures n'ont commencé que lorsque le diamètre des fruits était de l'ordre de $17 \mathrm{~mm}$. Cette phase a été représentée par un modèle monomoléculaire (fig 1A). La seconde phase caractérisée par la croissance finale de la pulpe a été représentée par un modèle logistique (fig 1B). Le choix de ces 2 modèles repose sur la description des courbes de croissance du noyau et de la pulpe de la pêche données par Lott (1932) et Dann et Jerie (1988). Le critère d'ajustement est celui des moindres carrés. La modélisation porte sur l'accroissement de diamètre $\left(D-D_{o}\right)$ observé entre la première mesure $\left(D_{o}\right)$ faite le 28 avril et les mesures successives $(D)$ réalisées jusqu'à la récolte du fruit (fig $1 C$ ). Les paramètres ont été estimés pour chaque fruit. Les courbes de vitesse de croissance (fig 1D) ont été calculées par dérivation du modèle. L'interprétation des cinq paramètres du modèle est présentée à la figure 1 . On en déduit que:

$P_{1}=$ la croissance cumulée lors de la première phase, $P_{2}{ }^{*} P_{1}=$ la vitesse de croissance en début d'étude,
$P_{3}=$ la croissance cumulée lors de la deuxième phase,

$P_{4} \times P_{3} / 4$ = la vitesse maximale de croissance pendant la deuxième phase,

$P_{5}=$ la date d'occurrence de cette vitesse maximale.

L'écart type des résidus de l'ajustement de chaque fruit a permis de juger de la qualité du modèle. Dans une optique d'utilisation du modèle à des fins de comparaison des courbes de croissance, nous avons vérifié que la variance d'estimation du paramètre $P_{i}$ des fruits étudiés était nettement inférieure à la variance du paramètre $P i$ dans la population. Le test de Wilks (Hand et Taylor, 1987) faisant intervenir les matrices de variance-covariance d'estimation des paramètres et leur matrice de variance-covariance dans la population a été réalisé de façon à juger si la variabilité d'estimation était suffisamment petite pour permettre une discrimination entre courbes de croissance.

\section{Comparaison des courbes de croissance}

La comparaison a porté, d'une part sur les courbes de croissance cumulée et d'autre part sur les courbes de vitesse de croissance. La période considérée pour cette comparaison est comprise entre le début de la période d'étude et le 26 juillet, date de récolte des premiers fruits. En procédant de la sorte, nous avons tronqué les courbes de croissance des fruits récoltés après le 26 juillet, mais ceci nous a semblé négligeable. En effet, la croissance réalisée après le 26 juillet n'a représenté en moyenne que $3 \%$ de la croissance totale des fruits et le diamètre mesuré le 26 juillet était très correlé à celui mesuré à la récolte $(R=$ 0,$95 ; P<0,01$ ).

La comparaison des courbes a été faite par ACP des 120 fruits décrits par les paramètres du modèle selon la procédure de Houllier (1987). Cette procédure facilite l'interprétation des différences observées entre courbes grâce à l'examen des corrélations entre paramètres du modèle et facteurs de l'ACP. Par ailleurs cette méthode présente plusieurs avantages techniques liés à la modélisation des courbes de croissance. En effet les vitesses de croissance sont aisément obtenues par dérivation du modèle, les intervalles entre mesures successives des diamètres peuvent être variables et les mesures manquantes influent peu sur les résultats. On a choisi pour l'ACP la distance entre fruits qui équivaut à la somme dans le temps des carrés des écarts entre leurs courbes (annexe 1). Cette distance accorde la même importance aux différentes périodes de la croissance. La métrique de l'ACP est calculée numériquement à partir de l'équation des courbes étudiées (annexe 1). Les courbes de croissance cumulée $(D-D o)$ et de vitesse de croissance $(d D / d t)$ des fruits ont été comparées séparément par deux ACP munies de leurs 2 métriques propres. L'analyse des fruits à l'aide des 2métriques est nécessaire car, compte tenu du critère de distance utilisé, des fruits peuvent être proches quant à leurs courbes de croissance cumulée mais lointains quant à leurs courbes de vitesse. 
A

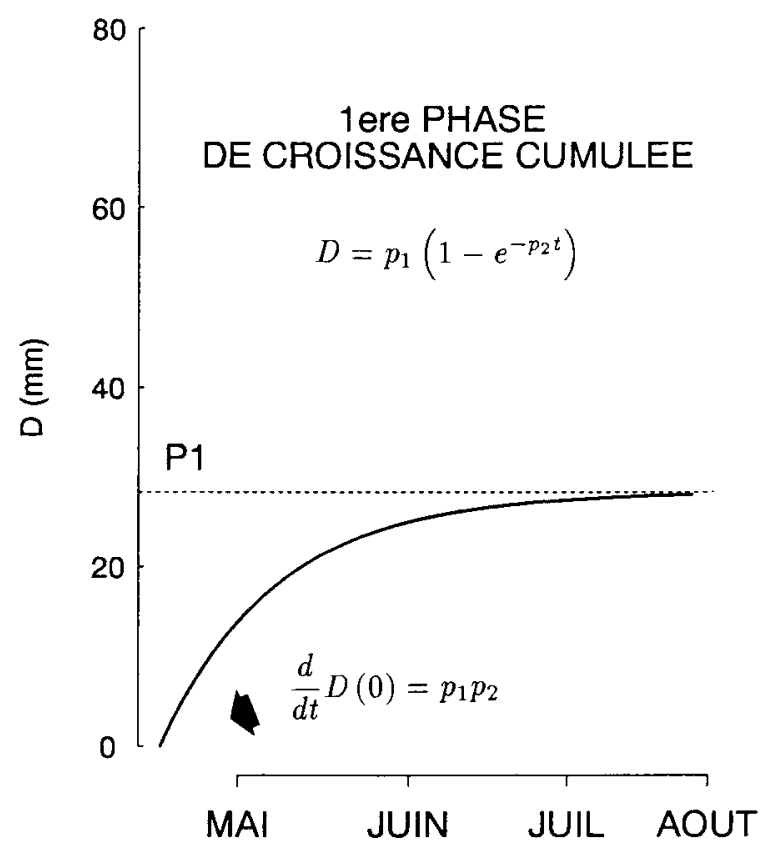

C

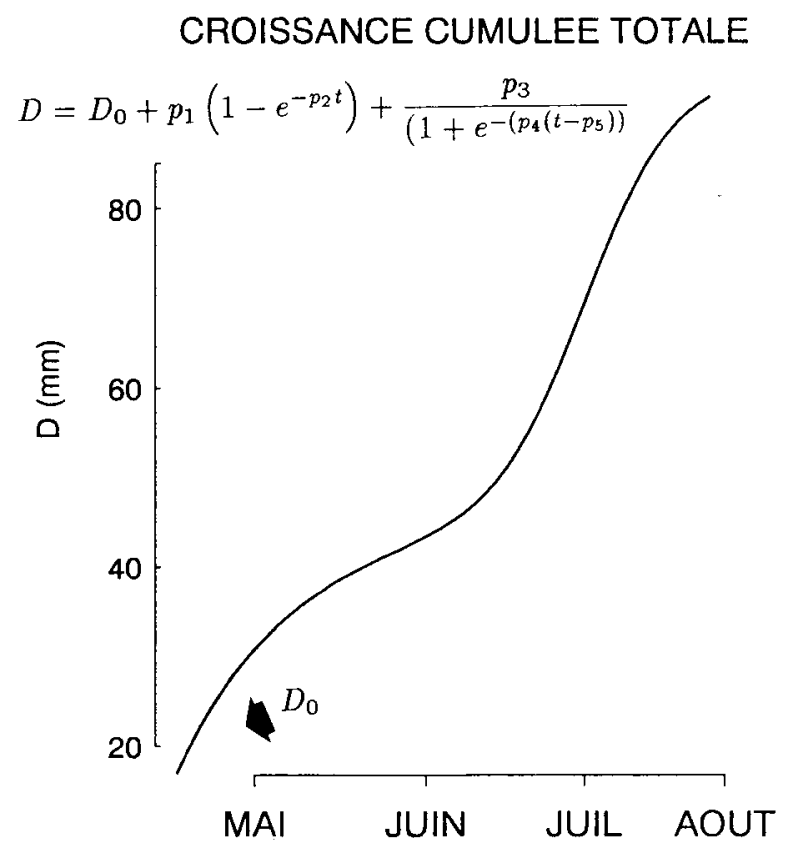

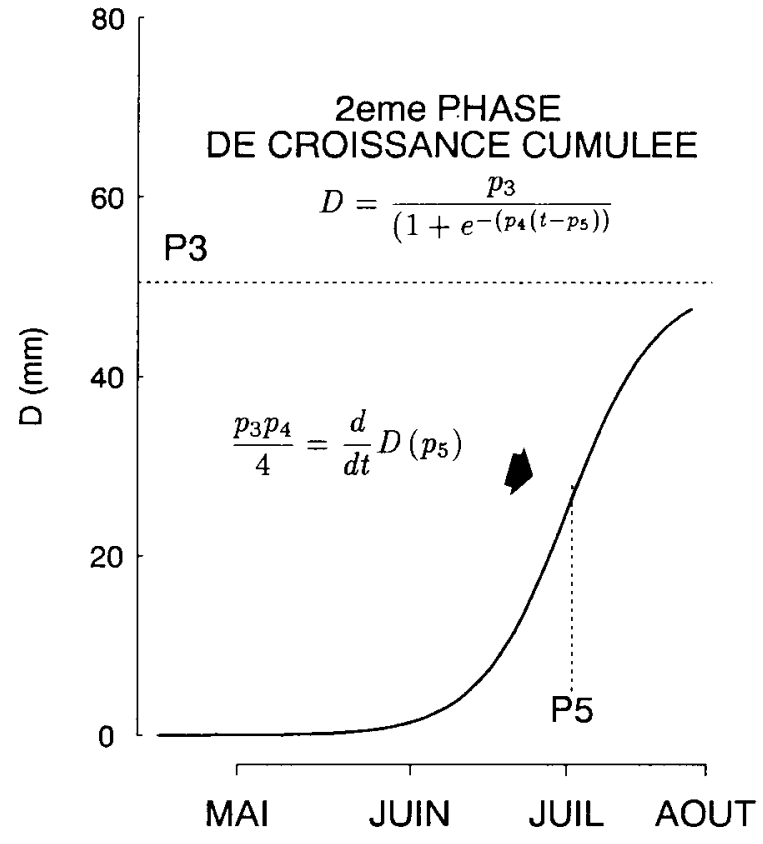

$\mathrm{D}$

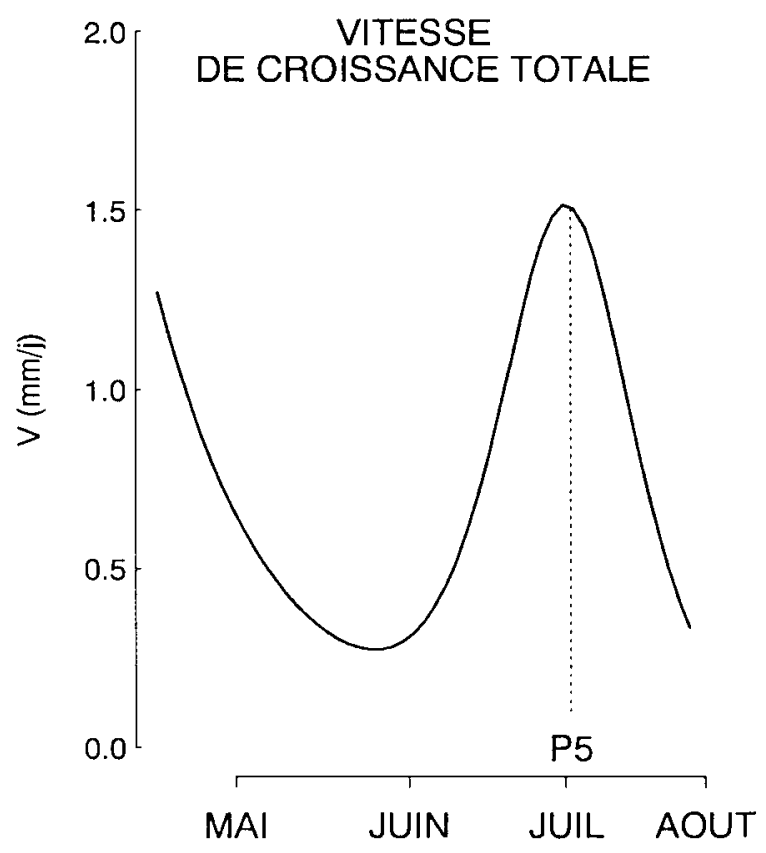

Fig 1. Modèle de croissance de la pêche : croissance lors de la $1^{\text {re }}$ phase (A); croissance lors de la $2^{e}$ phase (B); croissance cumulée totale (C); vitesse de croissance totale (D).

Une typologie des courbes de croissance a été construite par classification ascendante hiérarchique du moment d'ordre 2 d'une partition (Jambu, 1989), réalisée sur les coordonnées des fruits sur les premiers facteurs interprétables des ACP, comme cela est recommandé par Roux (1985). Une telle procédure supprime les distorsions induites par la multicolinéarité des variables (Converse et Morzuch, 1981 ; Maurer, 1986). En effet, la multicolinéarité entraîne des biais dans les résultats car les variables multicoli- 
néaires apportent plusieurs fois la même information à laquelle elles donnent un poids trop important. Le nombre de classes est choisi en se basant sur l'analyse des taux de variance des partitions associés aux différents niveaux de la hiérarchie (Jambu, 1989). Cette procédure permet de juger à partir de quel niveau dans la hiérarchie, la division en classes supplémentaires n'augmente plus la variabilité entre classes de façon notable.

\section{Comparaison de la qualité}

La comparaison de la qualité individuelle des 101 pêches a été faite par analyse factorielle des correspondances multiples ou AFCM (Benzécri, 1973; Jambu, 1989). Les 16 variables ont été découpées chacune en 5 modalités exclusives et d'effectifs équilibrés ( 80 modalités au total). La procédure de découpage des variables en modalités permet de prendre en compte les relations non linéaires entre variables, ce qui n'est pas le cas avec les variables quantitatives. La variable "sucre total», égale à la somme des variables "sucres réducteurs" et "saccharose" et classiquement utilisée dans les études concernant la qualité des fruits, a été mise en variable inactive ; il en est de même de la variable «jaune» car la part du fruit couverte par cette couleur est en général inférieure a $5 \%$, et du $\mathrm{pH}$ dont la variabilité entre fruits est de l'ordre de grandeur de l'erreur. On a retenu les facteurs qui extrayaient une part relativement importante de l'inertie, avaient des corrélations canoniques assez élevées et étaient interprétables. Les variables liées aux facteurs de l'AFCM sont celles ayant des rapports de corrélation élevés avec eux (Pialot et al, 1984).

Pour faciliter la présentation des résultats, les fruits ont été regroupés en classes de qualité. À cette fin une classification ascendante hiérarchique du moment d'ordre deux d'une partition a été réalisée sur les coordonnées des fruits sur les facteurs retenus à l'issue de I'AFCM.

La dispersion des points d'une classe de qualité a été représentée sur chaque plan factoriel sous hypothèse de normalité, par des ellipses d'inertie centrées sur le barycentre de ces points (Cornuet, 1982). Les ellipses de confiance bidimensionnelles à $P=95 \%$ des barycentres des classes ainsi que celles des modalités des variables sont représentées sur les plans factoriels sous hypothèse de normalité (Scheffé, 1959). Ce dernier calcul prend en compte la dispersion et l'effectif de la classe ou de la modalité considérée. II autorise la comparaison 2 à 2 des différents barycentres. L'interprétation est principalement basée sur la répartition de ces ellipses de confiance dans le plan.

\section{Relation entre croissance et qualité}

Nous nous sommes limités à l'étude de la relation entre qualité, telle que nous l'avons décrite par I'AFCM, et croissance. Aussi le tableau "qualité" sou- mis à l'analyse était celui reconstitué à partir des facteurs retenus lors de I'AFCM du tableau complet (Jambu, 1989). La procédure de reconstitution (annexe 2) fournit au biologiste l'information jugée pertinente qu'il souhaite effectivement mettre en relation avec des variables explicatives. Les variables explicatives considérées étaient les classes de courbes de croissance cumulée et de vitesse calculées à l'étape "Comparaison des courbes de croissance», ainsi que le diamètre initial et la date de maturité du fruit. Le diamètre initial du fruit (D0, fig 1) a été découpé en 5 modalités d'effectifs équilibrés et la date de maturité a été découpée en 3 modalités. Le découpage en classes permet comme précédemment de prendre en compte les relations non linéaires.

L'explication de la qualité par ces composantes de la croissance des fruits a été recherchée par analyse factorielle des correspondances sur variables instrumentales ou AFCVI (Ter Braak, 1986; Chessel et al, 1987). Cette méthode peut être considérée comme une généralisation de la régression au cas de 2 ensembles de variables, l'un étant explicatif de l'autre. L'AFCVI procède en 2 temps. Les variables de qualité du tableau reconstitué sont projetées dans l'espace défini par les variables de croissance, puis une AFC de ces projections est effectuée. Nous avons jugé de la pertinence de l'explication des variables de qualité par les variables de croissance en comparant l'ordre des modalités des variables et des classes de qualité sur les plans factoriels de l'AFCM et de l'AFCVI. Si les variables de croissance expliquent totalement la qualité des fruits, l'AFCM et l'AFCVI donnent les mêmes résultats. Le rapport de l'inertie totale du tableau "qualité» projeté, à celle du tableau initial fournit un indice global d'explication de l'information "qualité" par la croissance (Yoccoz, 1988). Il en est de même pour le rapport des variances des variables de qualité. Ces taux d'explication ne concernent que la partie de ces variables exprimée sur les facteurs de l'AFCM retenus pour la reconstitution. Les variables de qualité liées aux facteurs de l'AFCVI sont celles ayant de forts rapports de corrélation avec eux (Lebreton et al, 1988). L'interprétation des facteurs retenus a été basée sur l'examen de leurs corrélations avec les modalités des variables de croissance et sur celui des rapports de corrélation entre facteurs et variables de croissance. Les relations pertinentes ont ensuite été illustrées à l'aide d'une méthode robuste de régression locale (Chambers et al, 1983).

\section{RÉSULTATS}

\section{Modélisation et comparaison des courbes de croissance}

La variance expliquée par le modèle est comprise entre 98,2 et $99,9 \%$ selon les fruits. Les ajustements sont de bonne qualité puisque l'écart type des résidus est toujours inférieur à 
2,5 $\mathrm{mm}$ (fig 2). La variabilité entre fruits est surtout importante en fin de croissance (fig 3) et il n'y a pas de liaison nette entre le diamètre atteint pendant la première phase et celui mesuré à la récolte. La corrélation entre diamètre mesuré et diamètre à la récolte est faible en mai et juin $(R=0,12-0,28)$ et ne devient forte qu'après le 10 juillet $(R=0,6-0,9)$. La variance d'estimation des paramètres est de 3-6 fois plus faible que la variance des paramètres dans la population pour $P_{1}, P_{2}$ et $P_{4}$ et plus de 28 fois plus faible pour $P_{3}$ et $P_{5}$. Le seuil de signification de la statistique de Wilks correspond à une probabilité inférieure à $10^{-4}$. L'erreur d'estimation des paramètres est ainsi nettement inférieure à la variation de ceux-ci dans la population de fruits étudiée. L'utilisation de l'ACP sur les paramètres du modèle est donc possible. Les 2 premiers facteurs des ACP portant sur les courbes de croissance cumulées et de vitesse extraient respectivement 92 et $91 \%$ de la variabilité entre fruits. Les fruits s'ordonnent respectivement sur ces 2 plans factoriels selon leurs niveaux de croissance cumulée à l'issue de la première et de la deuxième phase de croissance et selon leur date et leur niveau de vitesse maximale lors de la deuxième phase. Sept classes de croissance cumulée et de vitesse ont été distinguées à l'issue de la classification faite sur les 2 premiers facteurs de chaque ACP (fig 4). Les classes de croissance et de vitesse ne sont que

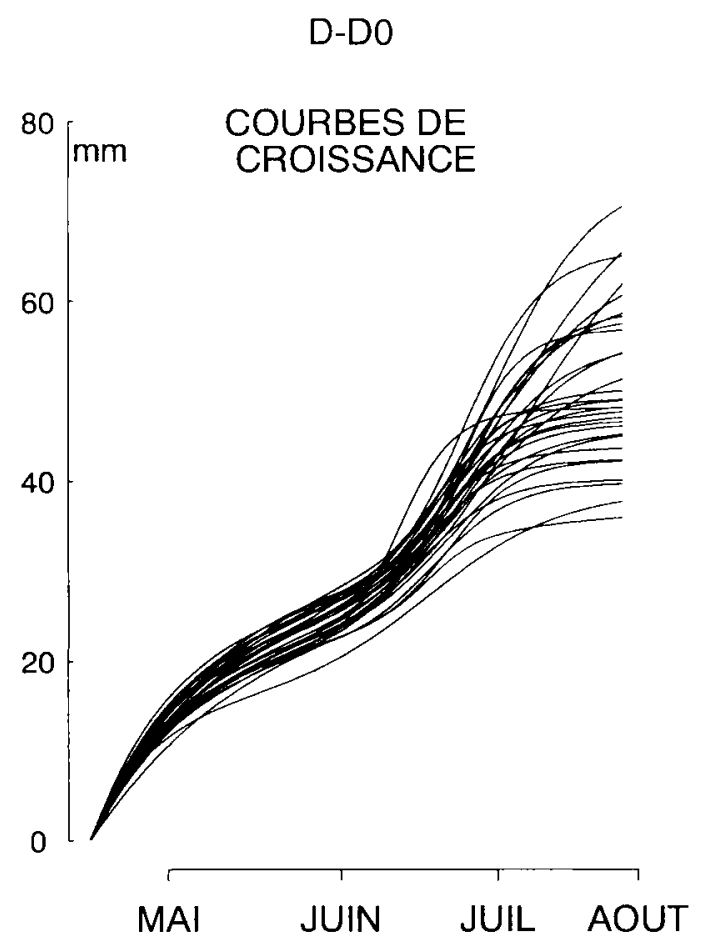

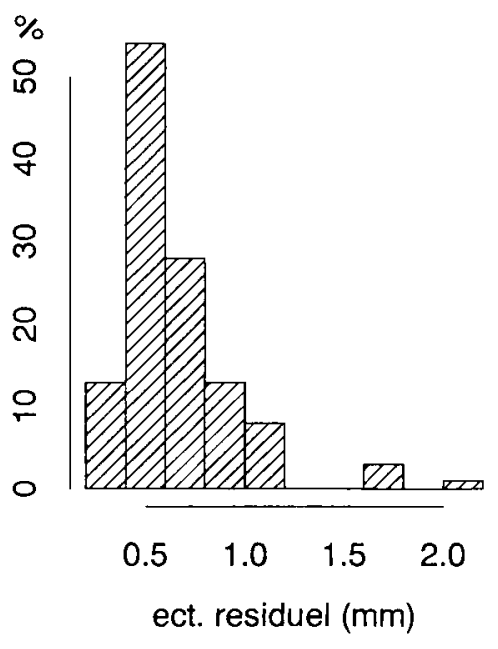

Fig 2. Distribution de l'écart type des résidus du modèle de croissance pour les 120 fruits étudiés.

faiblement liées entre elles et sont indépendantes des autres paramètres de la croissance que sont le diamètre initial du fruit et sa date de maturité.

\section{Variabilité de la qualité des fruits}

Les 3 premières corrélations canoniques des facteurs de I'AFCM ont des valeurs relativement

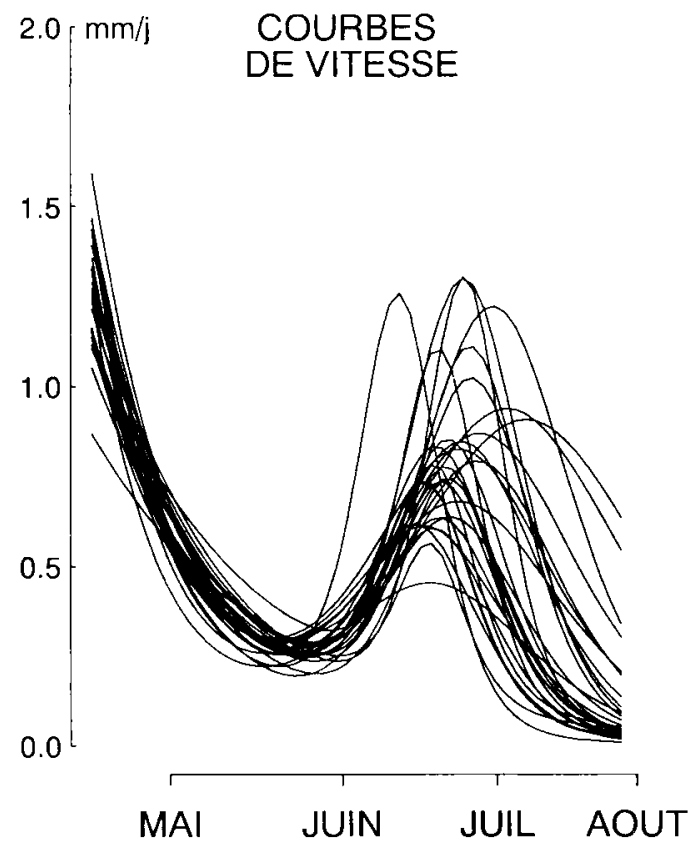

Fig 3. Courbes de croissance et de vitesse (échantillon de 30 fruits). 
D-DO

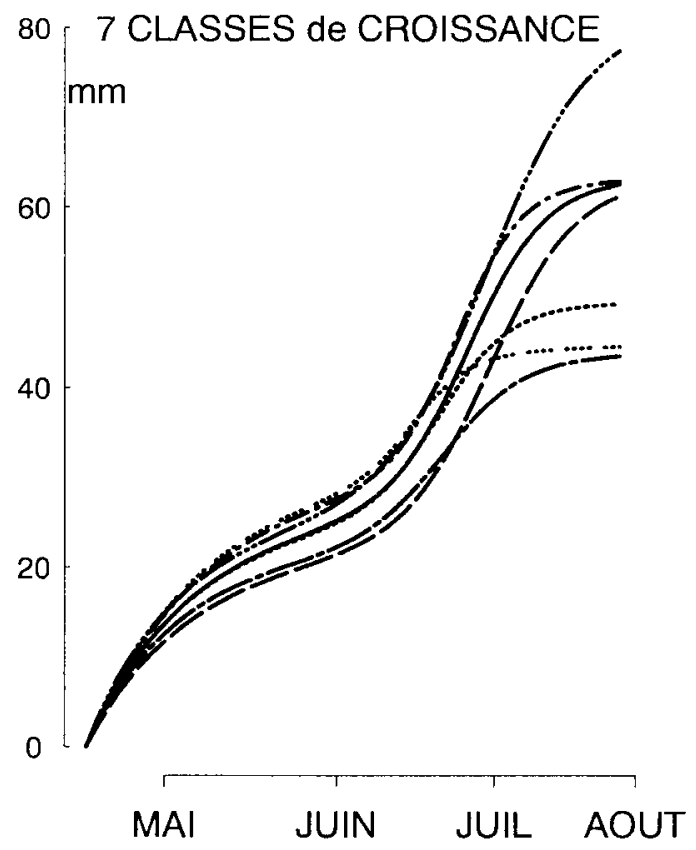

Fig 4. Classes de courbes de croissance et de vitesse.

élevées $(0,61,0,56$ et 0,49$)$, ce qui indique d'assez fortes différences de qualité entre fruits. Les axes de faible inertie n'ont pu être interprétés. Si l'on exclut le cas du pH qui a de faibles rapports de corrélation avec les facteurs, on distingue 2 familles de variables selon leurs rapports de corrélation avec les facteurs (tableau I) et selon l'ordre de leurs modalités sur les plans factoriels (figs 5 et 6 ).

La première famille constituée des variables de qualité gustative est surtout liée à $F_{1}$ qui peut être interprété comme un gradient d'augmentation de la teneur en saccharose et de la concentration en acide malique ainsi que de diminution de la teneur en sucres réducteurs et de la concentration en acide citrique. La teneur en sucre total et I'IR croissent également avec $F_{1}$ car l'augmentation de la teneur en saccharose est très supérieure à la diminution de celle en sucres réducteurs et de celle des autres matières solubles. La perception de l'acidité diminue avec $F_{1}$ car la somme des acides citrique et malique diminue (de 11,5-9 meq/100 g pour les groupes extrêmes sur l'axe) et le rapport acide malique / acide citrique augmente fortement (1-21). Cette évolution correspond à un gradient de qualité

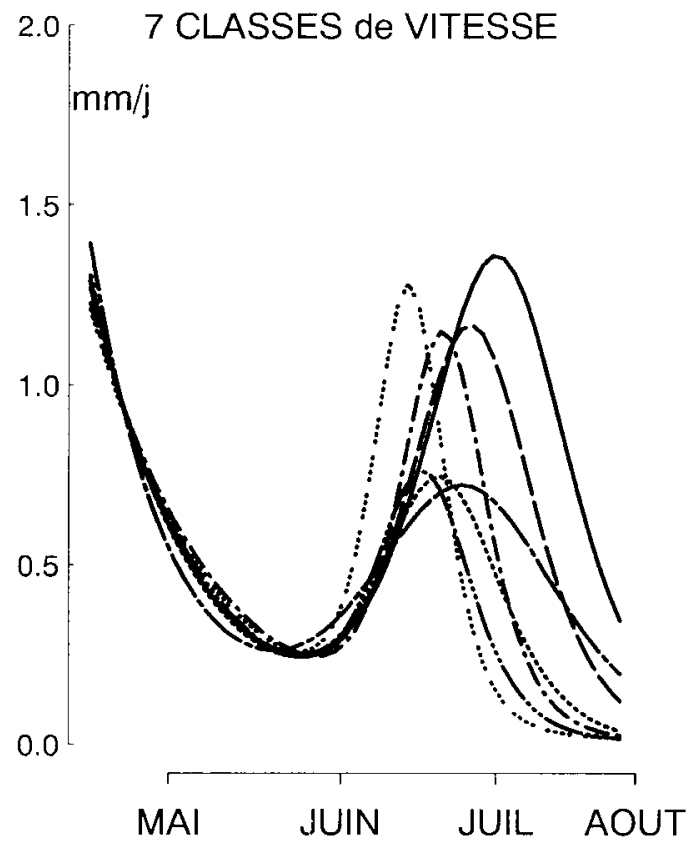

gustative opposant les fruits peu sucrés et acides aux fruits sucrés et doux. Ce gradient est en outre caractérisé par une diminution de la fermeté et une augmentation de la part de la couleur jaune. Les variables indice réfractométrique, sucres totaux, saccharose et acide citrique ont les rapports de corrélation les plus élevés avec $F_{1}$ et $F_{3}$. Ce sont elles qui contribuent le plus à la variabilité de la qualité gustative observée entre les fruits étudiés. Le groupe $\left(n^{\circ} 1\right)$ formé par ces 4 variables se différencie des variables de fermeté $\left(n^{\circ} 2\right)$ et de façon plus prononcée des variables "acide malique» et "sucres réducteurs" $\left(n^{\circ} 3\right)$.

La deuxième famille de variables concerne la coloration hormis le jaune. Elle est liée à $\mathrm{F}_{2}$, lequel décrit un gradient de coloration opposant les fruits peu lumineux, à faible chrominance, rouges violacés, aux fruits lumineux, à forte chrominance, rouges orangés.

Les fruits décrits par leurs coordonnées sur les 3 premiers facteurs de l'AFCM sont regroupés par classification en 4 classes. Elles représentent $39,26,23$ et $12 \%$ des pêches. La variabilité entre classes des valeurs des descripteurs de la qualité est importante (tableau II). 
Tableau I. Rapports de corrélation entre variables de qualité et facteurs pour l'analyse de la qualité (AFCM) et l'analyse de la relation qualité-croissance (AFCVI). Les rapports supérieurs à 0,30 sont en italique.

\begin{tabular}{|c|c|c|c|c|c|c|}
\hline & \multicolumn{3}{|c|}{$A F C M$} & \multicolumn{3}{|c|}{$A F C V I$} \\
\hline & $F 1$ & $F 2$ & F3 & F1 & $F 2$ & F3 \\
\hline $\mathrm{pH}$ & 0,21 & 0 & 0,06 & 0,12 & 0,05 & 0,01 \\
\hline Sucres totaux & 0,79 & 0,08 & 0,63 & 0,65 & 0,08 & 0,34 \\
\hline Saccharose & 0,87 & 0,12 & 0,66 & 0,71 & 0,14 & 0,32 \\
\hline IR & 0,84 & 0,08 & 0,63 & 0,65 & 0,08 & 0,34 \\
\hline Sucres réducteurs & 0,42 & 0,07 & 0,25 & 0,38 & 0,12 & 0,05 \\
\hline Acide citrique & 0,76 & 0,24 & 0,35 & 0,67 & 0,15 & 0,15 \\
\hline Acide malique & 0,32 & 0,10 & 0,12 & 0,31 & 0,15 & 0,06 \\
\hline Fermeté face colorée & 0,40 & 0,07 & 0,14 & 0,25 & 0,03 & 0,05 \\
\hline Fermeté face non colorée & 0,58 & 0,15 & 0,24 & 0,40 & 0,03 & 0,12 \\
\hline Couleur jaune & 0,31 & 0,12 & 0,03 & 0,32 & 0,05 & 0,06 \\
\hline Couleur orange & 0,06 & 0,42 & 0,07 & 0,12 & 0,18 & 0,06 \\
\hline Couleur rouge & 0,15 & 0,28 & 0,14 & 0,06 & 0,11 & 0,05 \\
\hline Couleur rouge violacé & 0,15 & 0,68 & 0,08 & 0,12 & 0,35 & 0,08 \\
\hline Luminance $\left(\mathrm{L}^{*}\right)$ & 0,18 & 0,72 & 0,16 & 0,04 & 0,13 & 0,01 \\
\hline Chrominance $\left(\mathrm{a}^{*}\right)$ & 0,02 & 0,63 & 0,23 & 0,04 & 0,25 & 0,05 \\
\hline Chrominance $\left(b^{*}\right)$ & 0,08 & 0,53 & 0,03 & 0,13 & 0,21 & 0,07 \\
\hline
\end{tabular}

\section{Relation entre croissance et qualité}

Nous avons soumis à I'AFCVI les variables de qualité reconstituées avec les 3 premiers facteurs interprétés de l'AFCM. Le tableau ainsi reconstitué conserve une structure analogue à celle du tableau initial (fig 7). Les variables explicatives utilisées sont le diamètre initial, les classes de courbes de croissance cumulées, les
Classes de vitesse et la date de maturité. Les corrélations canoniques des 3 premiers facteurs de l'AFCVI sont assez fortes $(0,54,0,34$ et 0,30$)$. La représentation des classes de qualité et des modalités des variables de qualité dans l'espace des 3 premiers facteurs de l'AFCVI est proche de celle observée sur les 3 premiers facteurs de l'AFCM et les interprétations des axes sont similaires (tableau I, figs 5 et 6 ). La part de l'inertie

Tableau II. Valeurs moyennes (et écarts types) des variables liées à la qualité pour chaque classe de qualité.

\begin{tabular}{|c|c|c|c|c|}
\hline Classes de qualité & 1 & 2 & 3 & 4 \\
\hline Sucres totaux $(g / 100 \mathrm{~g})$ & $5,08(1,12)$ & $6,82(1,75)$ & $7,59(1,39)$ & $11,75(1,14)$ \\
\hline Saccharose $(g / 100 \mathrm{~g})$ & $2,51(1,12)$ & $3,71(1,38)$ & $4,92(1,30)$ & $9,55(1,09)$ \\
\hline Sucres réducteurs $(\mathrm{g} / 100 \mathrm{~g})$ & $2,57(0,29)$ & $3,11(0,53)$ & $2,67(0,51)$ & $2,20(0,39)$ \\
\hline IR ( ${ }^{\circ}$ Brix) & $8,26(0,82)$ & $9,32(0,86)$ & $9,99(1,22)$ & $14,05(1,48)$ \\
\hline $\mathrm{pH}$ & $3,91(0,10)$ & $3,95(0,08)$ & $3,99(0,10)$ & $4,00(0,13)$ \\
\hline Acide malique ( $\mathrm{meq} / 100 \mathrm{~g}$ ) & $5,33(1,67)$ & $5,04(1,72)$ & $5,91(1,82)$ & $8,80(2,92)$ \\
\hline Acide citrique (meq/100 g) & $6,03(1,84)$ & $5,09(1,55)$ & $2,42(1,50)$ & $0,42(0,29)$ \\
\hline Fermeté face colorée $\left(\mathrm{kg} / \mathrm{cm}^{2}\right)$ & $1,83(2,73)$ & $1,17(0,97)$ & $0,72(0,21)$ & $0,62(0,39)$ \\
\hline Fermeté face non colorée $\left(\mathrm{kg} / \mathrm{cm}^{2}\right)$ & $2,20(3,23)$ & $1,44(1,00)$ & $0,83(0,25)$ & $0,60(0,17)$ \\
\hline Luminance $\left(\mathrm{L}^{*}\right)$ & $38,25(2,34)$ & $36,03(0,93)$ & $39,03(2,27)$ & $37,14(3,47)$ \\
\hline Chrominance $\left(a^{*}\right)$ & $12,75(5,00)$ & $5,73(3,14)$ & $15,88(5,76)$ & $12,28(5,35)$ \\
\hline Chrominance $\left(b^{\star}\right)$ & $11,70(3,33)$ & $4,45(1,36)$ & $13,79(3,33)$ & $11,34(3,59)$ \\
\hline Coloration jaune (\%) & $1,82(1,65)$ & $1,31(0,98)$ & $2,44(1,65)$ & $4,70(4,47)$ \\
\hline Coloration orange (\%) & $15,03(7,25)$ & $8,74(4,19)$ & $17,55(5,31)$ & $16,11(6,04)$ \\
\hline Coloration rouge $(\%)$ & $58,16(5,80)$ & $47,62(5,79)$ & $57,50(4,63)$ & $51,91(4,68)$ \\
\hline Coloration rouge violacé (\%) & $24,97(8,54)$ & $42,34(6,25)$ & $22,52(5,62)$ & $27,26(8,11)$ \\
\hline
\end{tabular}



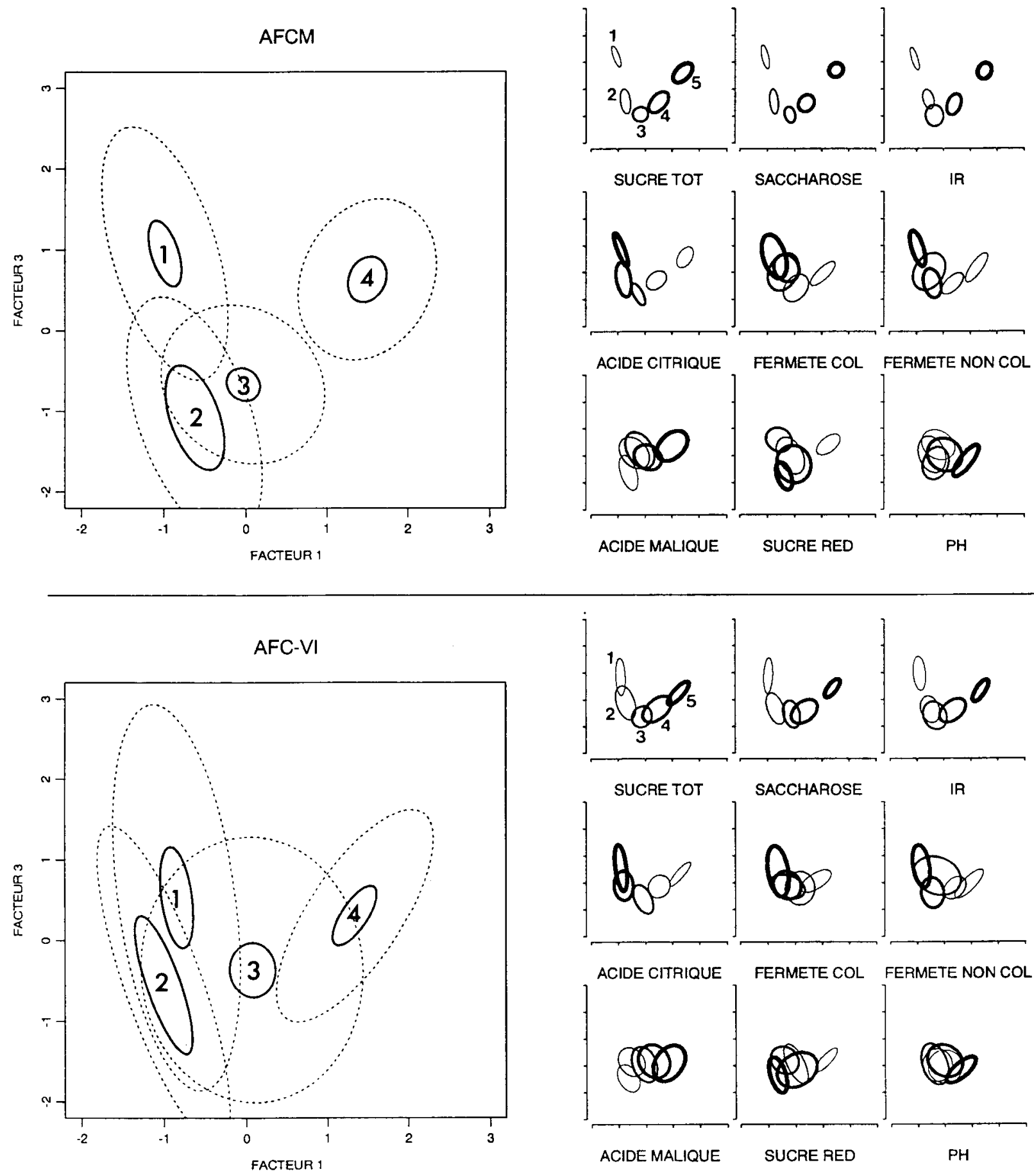

IR

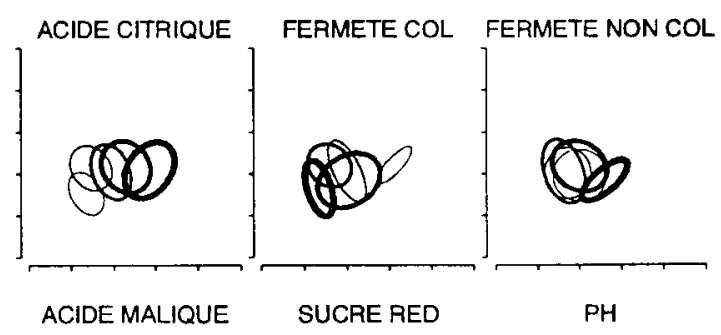

Fig 5. Ellipses d'inertie (pointillés) et de confiance de la moyenne (traits pleins) des 4 classes de qualité et des 5 modalités des variables de qualité gustative et de fermeté sur le plan F1 ${ }^{\star} F 3$ de l'AFCM et de l'AFCVI. La correspondance entre les figurés des ellipses et les niveaux des modalités est donnée pour la variable sucre total (modalité 1 = faible niveau de la variable; ...; modalité 5 = niveau élevé de la variable).

de la qualité (décrite par les 3 premiers facteurs de I'AFCM) expliquée par la croissance est de $54 \%$. Les variables de qualité gustative et de fermeté sont mieux expliquées que les variables de couleur (tableau III).

La variable diamètre initial n'explique pas la qualité car ses rapports de corrélation avec les facteurs de l'AFCVI sont faibles (tableau IV). Les variables décrivant les classes de courbes de croissance et de vitesse ont de forts rapports de corrélation avec $F_{1}$ et $F_{3}$ et donc avec la qualité gustative et la fermeté du fruit. L'examen des corrélations entre modalités de ces variables de croissance et facteurs montre que les fruits à 

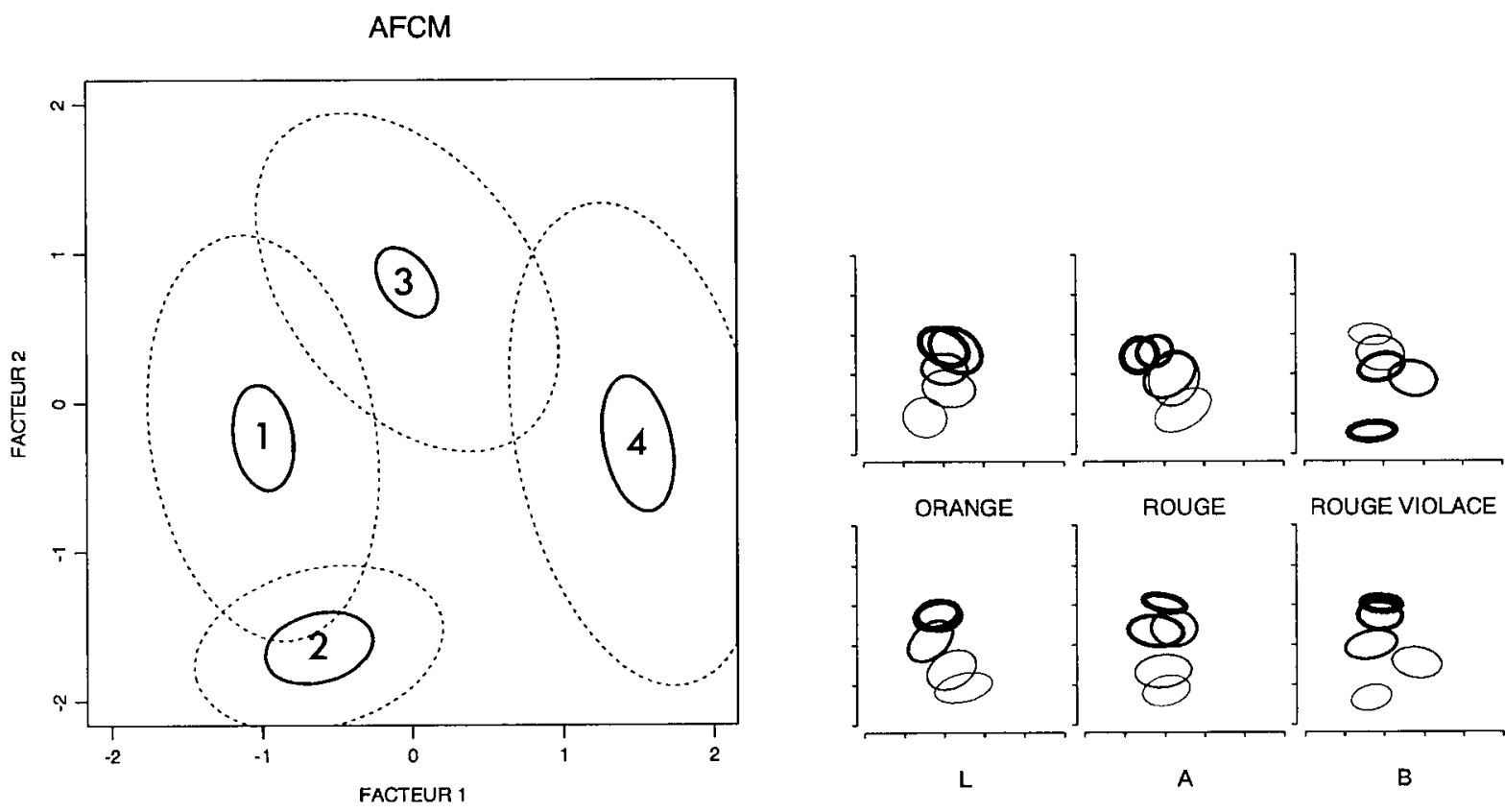

L

A
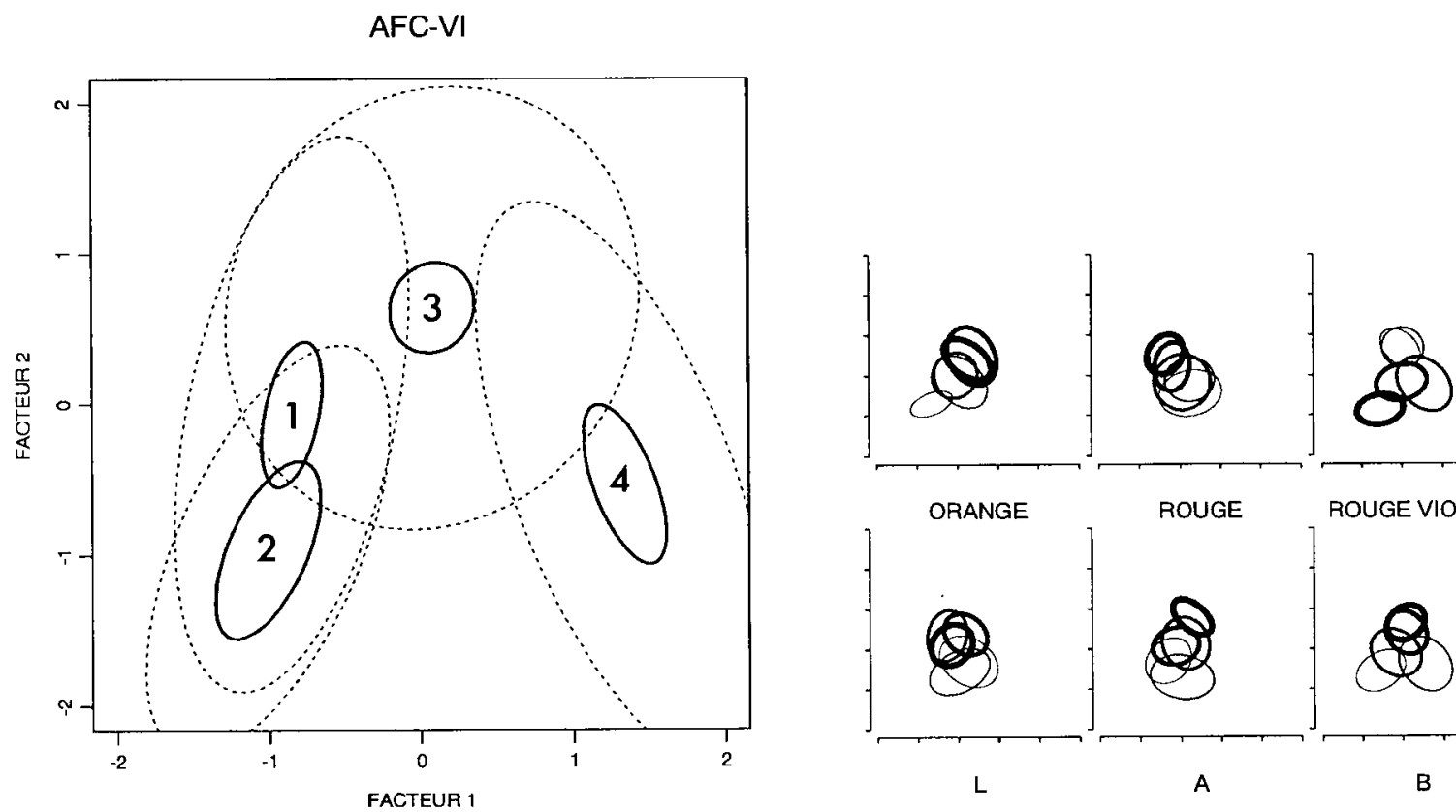

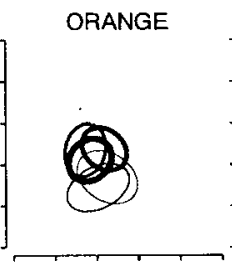

L

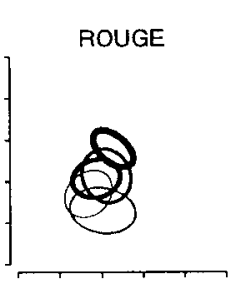

A

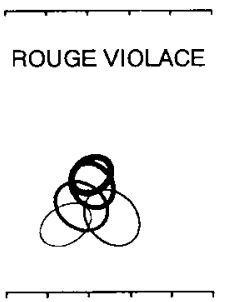

Fig 6. Ellipses d'inertie (pointillés) et de confiance de la moyenne (traits pleins) des 4 classes de qualité et des 5 modalités des va-

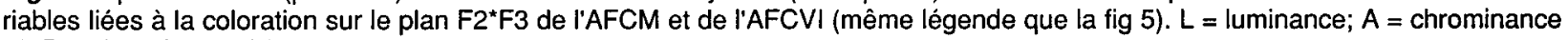
$a^{\star} ; B=$ chrominance $b^{*}$.

forte croissance cumulée et à forte vitesse maximale pendant la deuxième phase de leur croissance sont plus sucrés (teneur en saccharose élevée), moins acides (faible concentration en acide citrique) et moins fermes que les fruits ayant des caractéristiques de croissance opposées (fig 8). Ces fruits les plus sucrés ont en outre une date d'occurrence de leur vitesse maximale plus tardive (fig 8 ).

Les variables décrivant les classes de courbes de croissance, de vitesse et la date de maturité ont des rapports de corrélation assez élevés avec le deuxième facteur (tableau IV). La coloration rouge violacé semble augmenter lorsque la 
A

101 fruits

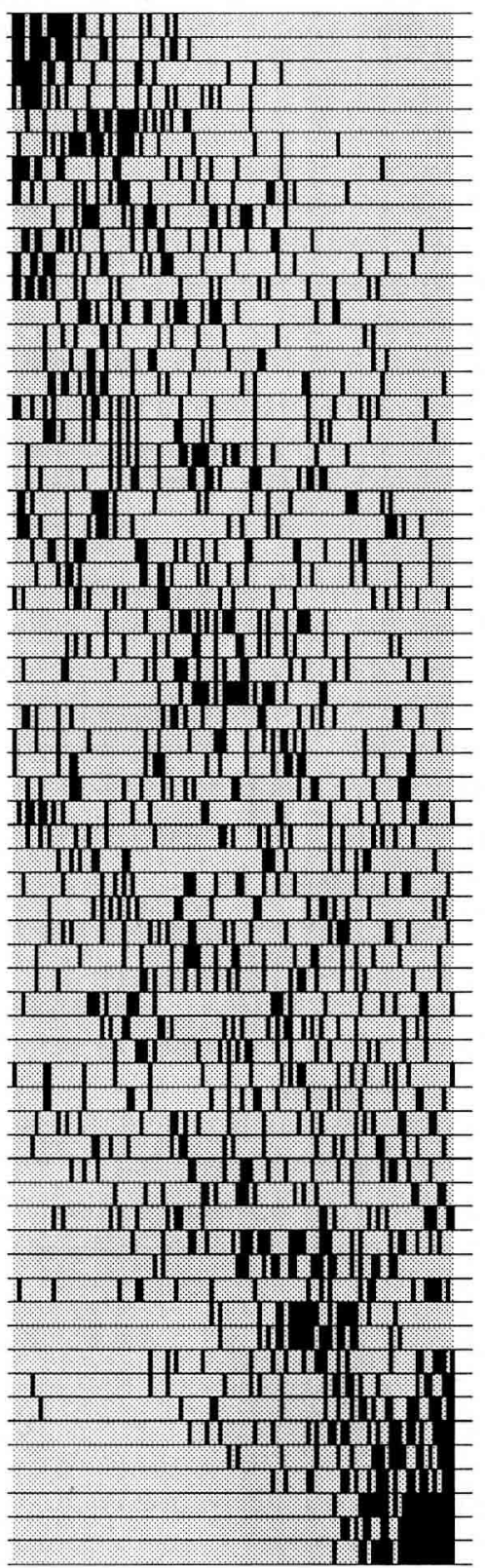

SACCH1 IR1
FERM NCO FERM NCOL5
ACIDE CITR5 SACCH2

ACIDE CITR4 FERM COL5 ACIDE MAL1 IR2

ROUGE5

SUCRE RED3

ORANGE1

FERM NCOL4

FERM NCOL3

FERM COL3

ACIDE MAL?

ROUGE VIOLS

IR3

SUCRE RED5

L3

D3

FERM COL4

ROUGE VIOL

a 4

ACIDE CITR3

ROUGE4

SUCRE RED

ACIDE MAL

L4

b4

L5

ROUGE VIOL4

SUCRE RED4 ROUGE VIOL2

a 1
ORANGE3

b5

as

FERM COL2

ACIDE MAL4

ROUGE3
ROUAL

12

ORANGE5

ROUGE2

a3

ORANGE4

L1

FERM NCOL2

ACIDE CITR2

ROUGE1

$\mathrm{SACCH}$

IR4

ROUGE VIOL3

b2

ACIDE MAL

FERM COL 1

SUCRE RED1

FERM NCOL

IR5

ACIDE CITR

SACCH5
B

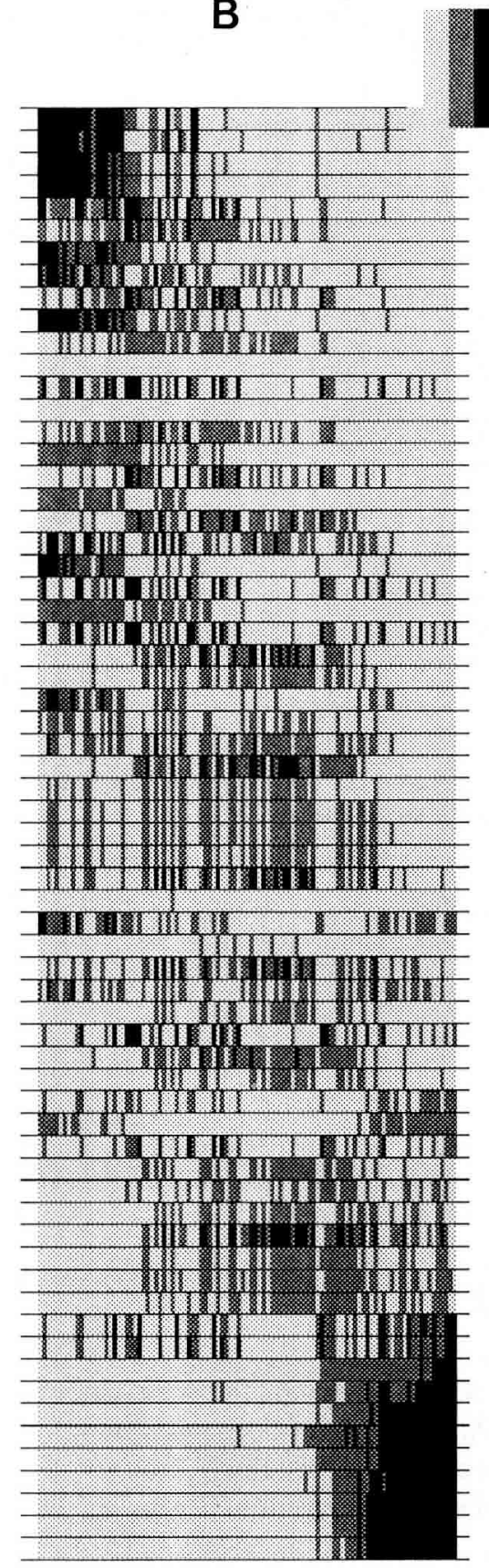

Fig 7. Tableaux des 101 fruits (colonnes) décrits par les variables de qualité découpées en 5 modalités numérotées de 1 à 5 dans les labels (lignes). Les lignes et les colonnes sont ordonnées selon le premier facteur de l'AFCM. Dans le tableau A des données brutes, en $0-1$, les trames noires indiquent les modalités associées aux fruits. Dans le tableau $B$ une gamme croissante de 3 grisés représente le niveau du codage reconstitué par les 3 premiers facteurs de l'AFCM (1: codage $<0,25 ; 2: 0,25 \leq$ codage $<0,45 ; 3$ : codage $\geq 0,45$ ). $S A C C H$ = saccharose; FERM NCOL = fermeté de la face non colorée; FERM COL = fermeté de la face colorée; CITR = acide citrique; $M A L=$ acide malique; $R E D=$ sucre réducteur; $V I O L=$ violacée.

maturité est précoce et lorsque le diamètre atteint lors de la première phase de développement augmente. La situation inverse est rencontrée pour la couleur orange et pour la chrominance. Les fruits ayant une date de vitesse de croissance maximale précoce ont une coloration rouge assez importante alors que l'orange domine dans le cas opposé (fig 9). La luminance ne semble pas liée à la croissance du fruit (fig 9). Les variables de croissance expliquent globalement bien la variabilité de coloration observée (fig 6), mais les liaisons sont faibles entre descripteurs de la croissance pris individuellement et variables de coloration (fig 9). 
Tableau Ill. Part de .. uriance des variables de qualité (actives dans I'AFC VI) expliquée par la croissance, en pourcentage.

Variable de qualité Part de la variable (\%)

$\begin{array}{ll}\text { Luminance }\left(\mathrm{L}^{*}\right) & 39 \\ \text { Chrominance }\left(\mathrm{a}^{*}\right) & 39 \\ \text { Chrominance }\left(\mathrm{b}^{*}\right) & 46 \\ \text { Couleur orange } & 49 \\ \text { Couleur rouge } & 42 \\ \text { Couleur rouge violacé } & 46 \\ \text { Sucres réducteurs } & 64 \\ \text { Saccharose } & 59 \\ \text { Acide malique } & 63 \\ \text { Acide citrique } & 62 \\ \text { Fermeté face colorée } & 60 \\ \text { Fermeté face non colorée } & 61 \\ \text { Indice réfractométrique } & 58\end{array}$

Tableau IV. Rapports de corrélation entre variables de croissance et facteurs de l'AFCVI.

\begin{tabular}{lccc}
\hline \multirow{2}{*}{ Variable de croissance } & \multicolumn{3}{c}{ Facteur de l'AFCVI } \\
& $F_{1}$ & $F_{2}$ & $F_{3}$ \\
\hline & & & \\
& & & \\
Diamètre initial & 0,03 & 0,12 & 0,07 \\
Vitesse & 0,92 & 0,46 & 0,48 \\
Croissance cumulée & 0,60 & 0,29 & 0,26 \\
Date de maturité & 0,12 & 0,36 & 0,21 \\
\hline
\end{tabular}

\section{DISCUSSION}

L'hypothèse d'une décomposition de la croissance en 2 phases permet de bons ajustements pour les courbes de croissance moyennes de pêches de différentes variétés (Baker et Davis, 1951; Dejong et Goudrian, 1989). Cette hypothèse convient également lorsque l'on modélise les croissances individuelles de fruits comme nous l'avons fait, puisque les ajustements observés étaient bons pour l'ensemble des fruits étudiés.

Dans notre échantillon, une part importante de la variabilité de la croissance s'exprime tardivement. Aussi, nous n'observons pas de relation entre le diamètre mesuré lors de la première phase de croissance (fin avril-juin) et le diamètre à la récolte, contrairement à ce qui a pu être calculé pour différentes variétés (Davis et Davis, 1948; Batjer et Westwood, 1958). Les vergers étudiés par ces auteurs étaient menés de façon à obtenir une croissance optimale. Le potentiel de croissance était probablement défini précocement lors de la multiplication cellulaire, les fruits les plus gros à la récolte étant ceux ayant le plus de cellules. Dans la situation que nous avons étudiée, l'optimum de croissance n'était pas recherché et une mauvaise alimentation de certains fruits était possible. Dans ce cas, il est probable que les différences dans l'élongation des cellules de la pulpe pendant la deuxième phase de croissance, conditionnent le plus le diamètre à la récolte. Les 2 phénomènes ne sont pas exclusifs puisque Proebsting (1962) montre que le diamètre à la récolte est déterminé par le diamètre mesuré précocement ainsi que par les conditions de culture susceptibles d'influer sur la croissance tardive du fruit.

La variabilité observée entre les classes de qualité est du même ordre que celle existant en moyenne entre différentes variétés (Souty et al, 1967; Souty et André, 1975; Delwiche et Baumgardner, 1983). Ceci montre l'importance d'une meilleure connaissance des facteurs sources de l'hétérogénéité de la qualité entre fruits. Hormis la couleur jaune qui ne représente qu'une faible part de la surface du fruit, il n'y a pas de liaison entre la coloration externe du fruit et sa fermeté ou sa qualité gustative. Les fruits étudiés s'ordonnent donc selon 2 gradients distincts. Le gradient de qualité gustative et de fermeté observé entre fruits rappelle l'évolution de celui classiquement rencontré pendant la maturation du fruit. En effet, on observe également lors de la maturation une diminution de la fermeté (Blake et al, 1931; Crochon, 1985), une augmentation de l'IR, des teneurs en sucres totaux, en saccharose (Nightingale et al, 1930; Lott, 1932; Crochon, 1985; Chapman et Horvat, 1990; Pavel et Dejong, 1990; Vizzotto et al, 1990) et de la concentration en acide malique (Chapman et Horvat, 1990) ainsi qu'une diminution des teneurs en sucres réducteurs (Pavel et Dejong, 1990; Vizzotto et al, 1990) et des concentrations en acide citrique (Chapman et Horvat, 1990). Tout se passe dans notre échantillon comme si la maturation des fruits les plus fermes et ayant une mauvaise qualité gustative à la récolte avait été stoppée précocement à un stade où la pulpe n'a pas encore atteint une composition biochimique satisfaisante.

Nos résultats montrent que la qualité gustative et la fermeté sont liées à la croissance du fruit pendant la deuxième phase. Plusieurs hypothèses explicatives peuvent être émises. Les sucres et les acides peuvent provenir directement des feuilles (Bollard, 1970; Ulrich, 1970) et 


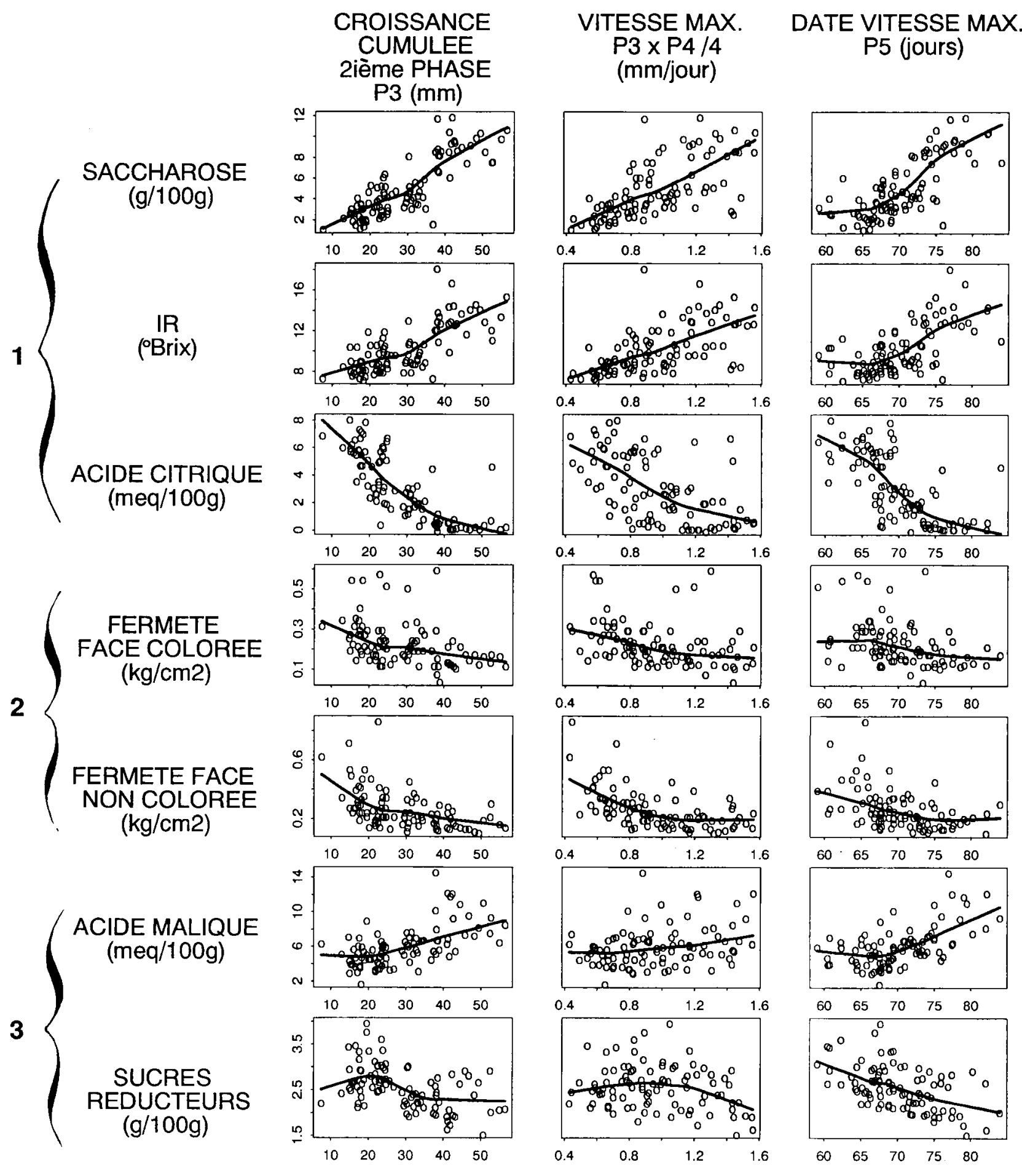

Fig 8. Relations entre croissance (absisses), qualité gustative et fermeté (ordonnées). Seules les variables de qualité actives dans l'analyse sont présentées; elles sont classées selon les groupes définis lors de l'étude de la variabilité de la qualité des fruits.

être stockés sans transformation dans le fruit. Une croissance élevée pendant la deuxième phase pourrait favoriser, par effet puits, l'apport externe de ces assimilats et ainsi permettre un important stockage de matières solubles. II faut supposer sous cette hypothèse que le transport de certains composés comme le saccharose, soit favorisé par rapport à celui d'autres comme l'acide citrique. Une seconde hypothèse explicative peut être que la production, pendant la deuxième phase, d'hormones liées à la croissance et notamment d'auxine, contenue en fortes concentrations dans la pulpe de la pêche, pourrait promouvoir la production d'éthylène (Miller et al, 1987) dont on connait l'action positive sur la maturation des fruits (Yang et Hoffman, 1984) et ainsi sur leur qualité gustative. Enfin une hypothèse explicative complémentaire peut être l'occurrence de conditions de milieu (température, insolation, etc) favorables à la 


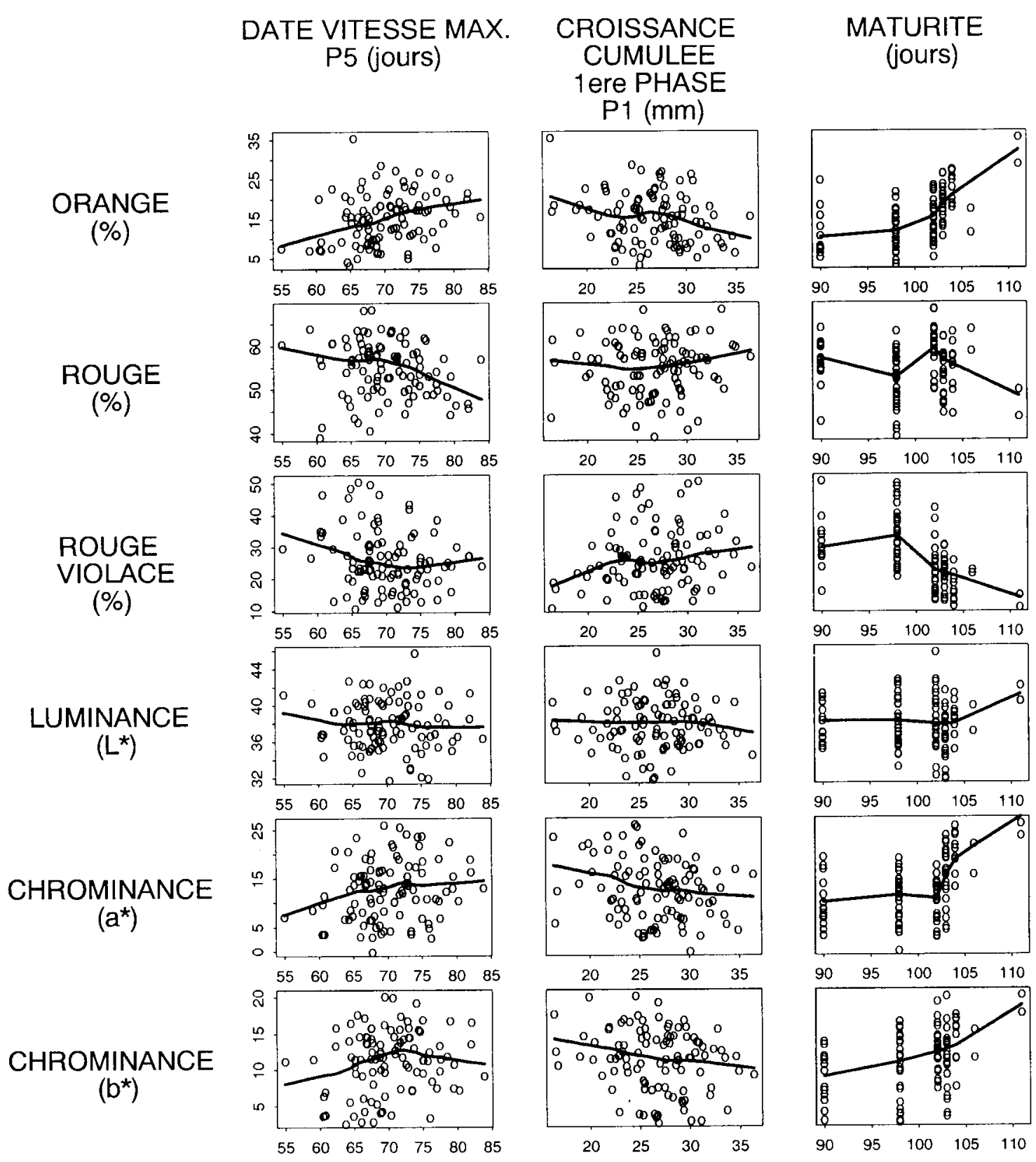

Fig 9. Relation entre croissance (abscisses) et coloration du fruit (ordonnées). Seules les variables de coloration actives dans l'analyse sont présentées.

croissance et aux réactions biochimiques internes au fruit pendant la deuxième phase sans qu'il y ait réellement de relation de dépendance entre la croissance et la qualité du fruit. Une meilleure compréhension des liaisons observées demanderait de décrire l'évolution des composés biochimiques lors de la croissance du fruit ainsi que les processus physiologiques liés à la croissance.

L'occurrence de fruits rouges violacés semble liée à l'importance de la croissance pendant la première phase. II est possible que les anthocyanes, pigments formés précocement dans le fruit (Saure, 1990) et responsables de la couleur rouge soient directement favorisés par cette croissance élevée car elles dépendent de la fourniture en glucides (Faust, 1965; Smock, 1966). Cependant les fruits rouges violacés sont également caractérisés par une date de maturité précoce qui pourrait être liée à un ensoleillement important, dont on connait l'action favorable sur la coloration rouge (Erez et Flore, 1986). Enfin nous avons noté que les fruits ayant une date de vitesse maximale de croissance tardive pendant la deuxième phase étaient plus oranges et moins rouges. Une interprétation complète de ces ré- 
sultats demanderait de mieux connaître le contrôle de la formation des anthocyanes et des caroténoïdes chez la pêche.

Ces résultats constituent un premier pas dans la connaissance des liaisons entre croissance et qualité du fruit. Ils devraient être testés dans d'autres situations (climat, variété, mode de culture).

\section{REMERCIEMENTS}

Ce travail a bénéficié d'une aide de la région PACA, convention $n^{\circ} 90 / 00760$.

Nous remercions $F$ Lescourret et $L$ Pages pour leurs conseils, $L$ Breuils et $M$ Reich pour leur participation aux analyses de qualité.

\section{ANNEXE 1}

L'analyse des paramètres des courbes de croissance proposée par Houllier (1986) procède en 2 étapes.

\section{Définition d'une métrique}

Nous disposons pour chacun des $n$ individus $i$, d'un modèle commun $Y(t, \Theta)$ fonction du temps $t$ et du vecteur des paramètres $\Theta$ ayant pour valeur $\Theta^{(i)}=\left(\theta_{1}(i), \ldots, \theta_{k}(i)\right)^{\prime}$. Sur la période d'étude $\left[t_{0}, t_{1}\right]$ nous utilisons une distance entre 2 individus $i$ et $j$ définie par

$\operatorname{dist}^{2}(i, j)=\frac{1}{t_{1}-t_{0}} \int_{t_{0}}^{t_{1}}\left(Y\left(t, \Theta^{(i)}\right)\right.$

$\left.-Y\left(t, \Theta^{(j)}\right)\left[t_{0}, t_{1}\right]\right)^{2} d t(1)$

Cette distance traduit une mesure de la surface entre 2 courbes.

L'approximation de $Y(t, \Theta(i))$ par un développement limité d'ordre un par rapport à $\Theta$ au voisinage du vecteur des paramètres moyens des $n$ individus $\bar{\Theta}=\left(\bar{\theta}_{1}, \ldots, \bar{\theta}_{k}\right)^{\prime}$ donne

$$
Y(t, \Theta(i))=Y(t, \Theta)+\sum_{I=1}^{k} \frac{\partial Y}{\partial \theta_{l}}(t, \Theta) \cdot\left(\theta,(i)-\theta_{l}\right)
$$

Reportant cette expression linéaire des paramètres dans (1) nous obtenons

$$
\operatorname{dist}^{2}(i, j) \approx(\Theta(i)-\Theta(i))^{\prime} M(\Theta(i)-\Theta(i))
$$

avec $\mathbf{M}$ matrice symétrique définie par

$$
M_{r s}=\frac{1}{t_{1}-t_{0}} \int_{t_{0}}^{t_{1}} \frac{\partial Y}{\partial \theta_{r}}(t, \bar{\theta}) \frac{\partial Y}{\partial \theta_{s}}(t, \bar{\Theta}) d t
$$

$r=1, k$ et $s=1, k$.

La matrice $M$ est définie positive sous des conditions de régularité suffisantes de la fonction $Y$. La distance entre 2 individus est donc exprimée à l'aide des paramètres associés à leurs modèles de croissance respectifs. L'application de cette méthode nécessite seulement le calcul numérique des éléments $M_{r s}$

\section{Analyse du tableau des paramètres}

Soit $P$ le tableau des paramètres centré sur la moyenne $\theta$ de l'échantillon. Chaque ligne de ce tableau représente un individu, chaque colonne les valeurs prises par un paramètre centré. Nous munissons les individus de la métrique $\mathbf{M}$ et les paramètres de la métrique usuelle $1 / n I, I$ étant la matrice identité. $\mathbf{V}=1 / n$ P'IP est la matrice de variance-covariance des paramètres. Effectuer l'ACP de $P$ revient à rechercher les valeurs propres $\lambda$ et vecteurs propres associés $u$ de VM sous la contrainte $\mathbf{u}^{\mathbf{M}} \mathbf{M u}=1$ (Lebart et al, 1982). Notons U la matrice dont les colonnes sont formées des vecteurs propres $\mathbf{u}$. Les coordonnées des individus sur les composantes principales sont données par les colonnes de PMU, et les corrélations des paramètres avec les composantes par S-1V MUL-1, $\mathbf{S}$ désignant la matrice diagonale des écarts types des colonnes de $\mathbf{P}$ et $\mathbf{L}$ la matrice diagonale des racines carrées des valeurs propres.

\section{ANNEXE 2}

\section{Reconstitution de tableau après AFCM}

Soit $X_{i j}$ l'élément de la $i \theta$ ligne et de la je colonne du tableau $X$ de $n$ lignes et $m$ colonnes soumis à une AFCM.

Si l'AFCM comporte $q$ facteurs on a (Jambu, 1989) :

$X_{i j}=\left(X_{i} X_{. j} / X_{.}\right) \times\left(1+\sum_{k=2, q} \mu_{k}^{-1 / 2} F_{k j} G_{k j}(1)\right.$ $\operatorname{avec} X_{i}=\sum_{j=1, m} X_{i j}$ 
$X_{. j}=\sum_{i=1, n} X_{i j}$

$X_{. .}=\sum_{i=1, n} \sum_{j=1, m} X_{i j}$

$\mu_{k}=$ valeur propre associée au $k^{e}$ facteur de I'AFCM.

$F_{k i}=$ coordonnées de l'individu $i$ sur le facteur $k$. $G_{k j}=$ coordonnées de la variable $j$ sur le facteur $k$.

Soit $p$ le nombre de facteurs retenus à l'issue de l'AFCM : la formule (1) se décompose en 2 termes :

$X_{i j}=X R_{i j}+\varepsilon_{i j}$

avec $X R_{i j}=\left(X_{i .} X_{. j} / X ..\right) \times\left(1+\sum_{k=2, p} \mu_{k}^{-1 / 2} F_{k i} G_{k j}\right)$

Le tableau $X \boldsymbol{R}$ formé des éléments $X R_{i j}$ est le tableau reconstitué à partir des $p$ premiers facteurs de l'AFCM. XR est la meilleure représentation de $\mathbf{X}$ dans un espace à $p$ dimensions.

\section{RÉFÉRENCES}

Baker GA, Davis LD (1951) Growth of the cheek diameter of peaches. Proc Amer Soc Hortic Sci 57, 104-110

Batjer LP, Westwood MN, (1958) Size of Elberta and $\mathrm{JH}$ Hale peaches during the thinning period as related to size at harvest. Proc Amer Soc Hortic Sci 72 , 102-105

Benzécri JP (1973) L'analyse des données. II. L'analyse des correspondances. Dunod, Paris

Bittner DL, Manning $J$ (1967) Automated neocuproïne glucose method: critical factors and normal values. In: Automation in analytical chemistry (Technicon corp, eds) Ardsley, New York, 33-36

Blake MA, Davidson OW, Addoms RM, Nightingale GT (1931) Development and ripening of peaches as correlated with physical characteristics, chemical composition, and histological structure of the fruit flesh : I. Physical measurement of growth and flesh texture in relation to the market and edible qualities of the fruit. $N J$ Agric Exp Stn Bul 525, 1-35

Boehringer (1988) Méthodes enzymatiques pour l'analyse agro-alimentaire. Fiches descriptives d'analyses. Boehringer, Mannheim, France

Bollard EG (1970) The physiology and nutrition of developping fruits. In: The biochemistry of fruits and their products (AC Hulme, ed) Acad Press, Londres, New York, 1, 387-425

Chambers JMB, Cleveland WS, Kleiner B, Tukey PA (1983) Graphical methods for data analysis. Wadsworth and Brooks/Cole Publishing Company, Pacific Grove, California

Chapman GW, Horvat RJ (1990) Changes in nonvolatile acids, sugars, pectin, and sugar composition of pectin during peach ( $\mathrm{Cv}$ Monroe) maturation. $J$ Agric Food Chem 38, 383-387
Chessel D, Lebreton JD, Yoccoz N (1987) Propriétés de l'analyse canonique des correspondances : une illustration en hydrobiologie. Rev. Stat App/ 35, 5572

Converse KA, Morzuch B.J. (1981) A descriptive model of snowshoe hare habitat. In: The use of multivariate statistics in studies of wildlife habitat (DE Capen, ed) USDA Forest Service, General Technical Report RM 87, 232-241

Cornuet JM (1982) Représentation graphique de populations multinormales par des ellipses de confiance. Apidologie 13, 15-20

Crochon M (1985) Quality of peaches as a function of picking time and consumer's preferences. Acta Hortic 173, 433-440

Dann IR, Jerie PH (1988) Gradients in maturity and sugar level of fruit within peach trees. J Am Soc Hortic Sci 113, 27-31

Davis LD, Davis M M (1948) Size in canning peaches. The relation between the diameter of cling peaches early in season and at harvest. Proc Am Soc Hortic Sci 51, 225-230

Dejong TM, Goudrian J (1989) Modeling peach fruit growth and carbohydrate requirements: reevaluation of double-sigmoid growth pattern. J Am Soc Hortic Sci 114, 800-804

Delwiche MJ, Baumgardner RA (1983) Ground color measurements of peach. J Am Soc Hortic Sci 108, 1012-1016

Doud DS, Ferree DC (1980) Influence of altered light level on growth and fruiting of mature 'delicious' apple trees. J Am Soc Hortic Sci 105, 325-328

Erez A, Flore JA (1986) The quantitative effect of solar radiation on 'Redhaven' peach fruit skin color. Hortscience 21, 1424-1426

Faust M (1965) Physiology of anthocyanin development in Mclntosh apple II. Relationship between protein synthesis and anthocyanin development. Proc Am Soc Hortic Sci 87, 10-20

Gaillard JP, Cassin J, Arias N, Ciccoli H (1976) Contribution à l'étude de l'échantillonnage des agrumes. I-Monographie de la récolte d'un clémentinier. Fruits 31, 31-59

Hand DJ, Taylor CC (1987) Multivariate analysis of variance and repeated measures. Chapman \& Hall, Londres

Holyoake J (1989) A vision system for microcomputer, Microscale IC, version 1.1. Digithurst Ltd

Houllier F (1987) Comparaison des courbes et modèles de croissance: choix d'une distance entre individus. Stat Anal Données 12, 17-36

Jambu M (1989) Exploration informatique et statistique des données. Dunod, Paris

Jones ID (1931) Preliminary report on relation of soil moisture and leaf area to fruit development of Georgia Belle peach. Proc Am Soc Hortic Sci 28, 6-14

Kaps ML, Cahoon GA (1989) Berry thinning and cluster thinning influence vegetative growth, yield, fruit 
composition, and net photosynthesis of Seyval blanc grapes. J Am Soc Hortic Sci 114, 20-24

Lai R, Woolley DJ, Lawes GS (1990) The effect of inter-fruit competition, type of fruiting lateral and time of anthesis on fruit growth of kiwifruit (Actinidia deliciosa). J Hortic Sci 65, 87-96

Lebart L, Morineau A, Fénelon JP (1982) Traitement des données statistiques. Dunod, Paris

Lebreton JD, Chessel D, Richardot-Coulet M, Yoccoz $N$ (1988) L'analyse des relations espèces-milieu par l'analyse canonique des correspondances. II. Variables de milieu qualitatives. Acta Oecol Oecol Gen 9, 137-151

Lott RV (1932) The growth rate and chemical composition of the Hiley peach from stone formation to flesh maturity. Proc Am Soc Hortic Sci 29, 1-7

Magness JR (1928) Relation of leaf area to size and quality in apples. Proc Am Soc Hortic Sci 25, 285288

Marini RP, Trout JR (1984) Sampling procedures for minimizing variation in peach fruit quality. $J$ Am Soc Hortic Sci 109, 361-364

Maurer BA (1986) Predicting habitat quality for grasslands birds using density-habitat correlations. $J$ Wild Manage 50, 556-566

Miller AN, Walsh CS, Cohen JD (1987) Measurement of Indole-3-acetic acid in peach fruits (Prunus persica L Batsch cv Redhaven) during development. Plant Physiol 84, 491-494

Monestiez P, Habib R, Audergon JM (1989) Estimation de la covariance et du covariogramme pour une fonction aléatoire à support arborescent : application à l'etude des arbres fruitiers. In: Geostatistics (M Armstrong, eds) Kluwer Acad Publ, 1, 39-56

Monet R (1983) Le pêcher, génétique et physiologie. Masson, Paris

Nightingale GT, Addoms RM, Blake MA (1930) Development and ripening of peaches as correlated with physical characteristics, chemical composition, and histological structure of the fruit flesh: III. Macrochemistry. N J Agric Exp Stn Bull 494, 2-15

Overholser EL, Claypool LL. (1931) The relation of leaf area per peach to physical properties and chemical composition. Proc Am Soc Hortic Sci 28, 15-17

Patten KD, Patterson ME, Proebsting EL (1986) Factors accounting for the within-tree variation of fruit quality in sweet cherries. Hortscience 21, 356-360

Pavel EW, Dejong TM (1990) Seasonal growth patterns and carbohydrate economy of peach fruits. In: XXIII Int Hortic Congr, Abstracts of contributed papers. 1. Oral. Florence, Italie, 565

Pialot D, Chessel D, Auda Y (1984) Description de milieu et analyse factorielle des correspondances multiples. CR Séances Acad Sci Paris 298, 309-314
Proebsting EL (1962) Factors influencing the relationship of harvest diameter to reference date diameter of Elberta peaches. Proc Am Soc Hortic Sci 80, 154162

Proebsting EL (1990) The interaction between fruit size and yield in sweet cherry. Fruit Var J 44, 169172

Reynolds AG (1989) Riesling grapes respond to cluster thinning and shoot density manipulation. $J \mathrm{Am}$ Soc Hortic Sci 114, 364-368

Roper TR, Loescher WH (1987) Relationships between leaf area per fruit and fruit quality in 'Bing' sweet cherry. Hortscience 22, 1273-1276

Roux M (1985) Algorithmes de classification. Masson, Paris

Saure MC (1990) External control of anthocyanin formation in apple. Sci Hortic 42, 181-218

Scheffé H (1959) The analysis of variance. John Wiley \& Sons, New York

Smock RM (1966) Laboratory studies of anthocyanin development in Mclntosh apples. Proc Am Soc Hortic Sci 88, 80-88

Souty M, André P (1975) Composition biochimique et qualité des pêches. Ann Technol Agric 24, 217-236

Souty M, Perret A, André P (1967) Premières observations sur quelques variétés de pêches destinées à la conserve. Ann Technol Agric 16, 55-68

Ter Braak CJF (1986) Canonical correspondence analysis: a new eigenvector technique for multivariate direct gradient analysis. Ecology 67, 11671179

Ulrich R (1970) Organic Acids. In: The biochemistry of fruits and their products (AC Hulme) Acad Press, Londres, New York, 1, 89-118

Vizzoto G, Pinton R, Costa G, Maggioni A (1990) Sugar content and metabolism in developping peach fruit (Prunus persica L. Batsch). In: XXIII Int Hortic Congr, Abstracts of contributed papers. 2. Poster. Florence, Italie, 3284

Weinberger JH (1931) The relation of leaf area to size and quality of peaches. Proc Am Soc Hortic Sci 28, 18-22

Weinberger JH, Cullinan FP (1932) Further studies on the relation between leaf area and size of fruit, chemical composition, and fruit bud formation in Elberta peaches. Proc Am Soc Hortic Sci 29, 23-27

Yang SF, Hoffman NE, (1984) Ethylene biosynthesis and its regulation in higher plants. Annu Rev Plant Physiol 35, 155-189

Yoccoz NG (1988) Le rôle du modèle euclidien d'analyse des données en biologie évolutive. Thèse, Univ de Lyon

Zuconi F (1986) Peach. In: Handbook of fruit set and development (SP Monselise, eds) Boca Raton, 303-321 